\title{
LEAST SQUARES APPROXIMATIONS FOR DUAL TRIGONOMETRIC SERIES
}

\author{
by ROBERT B. KELMAN† and CHESTER A. KOPER, Jr
}

(Received 15 November, 1971)

1. Introduction. A systematic and easily automated least squares procedure, not using integral equations or special functions, is presented for approximating the solutions of general dual trigonometric equations. This is desirable, since current analytic methods apply only to special equations, require the use of integral equation and special function theory, and do not lend themselves easily to numerical work; see, e.g. $[1,2,6,8,9,10,11,12,13,14,15,16,17]$.

The series are described in $\S 2$. The equation for least squares approximation is derived in $\S 3$ and used to develop the computer program DUTSA (DUal Trigonometric Series Analyser). A few examples are presented in $\S 4$ from amongst the several dozen dual trigonometric series to which DUTSA has been applied. These include examples from classes of dual equations for which solutions are not now available (save possibly for very special cases), e.g. arbitrary series not connected with applications, series associated with harmonic mixed boundary value problems in (bounded) rectangles and series with one of the mixed boundary conditions corresponding to a (linear) radiation condition. $\neq$ The evidence from these computations indicates that most dual trigonometric series from applications can be solved with a relative least squares error (defined in $\S 5$ ) smaller than $4 \%$ in 10 seconds or fewer on a computer with a 6 microsecond multiplication time.

Our analysis, in common with earlier studies, is heuristic: the numerical evidence is suggestive, but it does not rigorously describe the limitations of the method. The least squares approach is so simple that we searched the literature carefully, but found no evidence of its prior use. We expect that it will be helpful in studying other dual and similar series.

2. Series and expansions. Let $\left\{\phi_{n}(x): n=1,2, \ldots\right\}$ be a complete set of orthonormal, trigonometric functions associated with the Sturm-Liouville operator $d^{2} / d x^{2}$ on the interval $[0, d]\left[4\right.$, pp. $231 \mathrm{ff}$., p. 271], and $c$ a fixed point in the interval. $\left\{b_{n 1}\right\}$ and $\left\{b_{n 2}\right\}$ are sequences of positive constants. The sequence of functions $\left\{b_{n}(x)\right\}$ is defined on $[0, d]$ by $b_{n}(x)=b_{n 1}$ for $x<c$ and $b_{n}(x)=b_{n 2}$ for $x>c$. Any series $\sum_{n} a_{n} b_{n}(x) \phi_{n}(x)$ (abbreviation: $S(a)$ ) constructed formally with an arbitrary sequence of real numbers, $a=\left\{a_{n}\right\}$, is called a dual trigonometric series. $S(a)$ is called the dual trigonometric expansion of a function $f(x)$ if the relation

$$
\sum_{n=1}^{\infty} a_{n} b_{n}(x) \phi_{n}(x)=f(x)
$$

$\dagger$ The work of RBK was supported in part by Contract No. N00014-67-A-0299-0001 (Task No. N0041-278) with the Office of Naval Research (U.S.A.).

$\ddagger$ The definition of radiation boundary condition is given in $[11, \S 1.2]$. A theoretical solution for dual trigonometric series associated with a problem in which the potential satisfies a radiation condition over part of the boundary has been suggested in [11, p. 151], but this did not include the radiation condition as one of the mixed conditions. 
holds on $[0, d]$ (in an ordinary or generalized sense). The sequences $\left\{\phi_{n}\right\}$ and $\left\{b_{n}\right\}$ are referred to, respectively, as the kernel and the modifier of the series. The general problem is: given $\left\{\phi_{n}\right\},\left\{b_{n}\right\}$ and $f$, find $\left\{a_{n}\right\}$.

3. Least squares approximation. An approximation of the form

$$
a^{N}=\left(a_{1}^{N}, a_{2}^{N}, \ldots, a_{N}^{N}, 0,0, \ldots\right)
$$

for $a$ in equation (1) is sought to minimize the $L_{2}$ norm of the deviations, i.e., $a^{N}$ is the vector minimizing

$$
\|S(a)-f\|^{2}=\int_{0}^{d}\left[\sum_{n=1}^{\infty} a_{n} b_{n}(x) \phi_{n}(x)-f(x)\right]^{2} d x
$$

subject to the restraint $a_{n}=0$ for $n>N$. This necessitates that

$$
\frac{\partial}{\partial a_{n}}\|S(a)-f\|^{2}=0 \quad(n=1,2, \ldots, N) .
$$

Equation (2) determines $a^{N}$ and implies that

$$
\begin{gathered}
\sum_{n=1}^{N} A_{k n} a_{n}^{N}=F_{k} \quad(k=1,2, \ldots, N), \\
A_{k n}=\int_{0}^{d} \phi_{k}(x) \phi_{n}(x) b_{k}(x) b_{n}(x) d x, \\
F_{k}=\int_{0}^{d} f(x) b_{k}(x) \phi_{k}(x) d x .
\end{gathered}
$$

Program DUTSA implements equations (3), (4), (5). The user supplies as data $c, d$, and $N$. The subroutine for $A_{k n}$ is based upon expressing the integrals in equation (4) as a linear sum of cosines. Either (i) the integrals in equation (5) are computed beforehand and read in as data or (ii) $f$ is provided as a function subroutine and the integrals are computed automatically by Filon integration [3, p. 194]. Gaussian elimination is used as an efficient method for solving (3), since the matrix $\left(A_{k n}\right)$ is not sparse [5].

The plotting of the figures below was done on line by a CDC 3898 microfilm recorder and controller. All computations were perfomed on a CDC 6400 .

\section{Examples.}

Example 1. This problem occurs in fracture mechanics and was brought to our attention 
by our colleague, $\operatorname{Dr} F$. W. Smith. Because of the nonstandard form of the modifier, it has not, to our knowledge, been solved previously.

$$
\begin{aligned}
& \frac{a_{1}}{\sqrt{ }}+\sum_{n=2}^{\infty} a_{n}\left(\frac{2}{\pi}\right)^{1 / 2} \cos (n-1) x=\pi \cos 2 x \quad(0<x<\pi / 4), \\
& \frac{a_{1}}{2 \sqrt{ } \pi}+\sum_{n=2}^{\infty} \frac{a_{n}}{\sqrt{4+(n-1)^{2}}}\left(\frac{2}{\pi}\right)^{1 / 2} \cos (n-1) x=0 \quad(\pi / 4<x<\pi) .
\end{aligned}
$$

Plots of $S\left(a^{20}\right), S\left(a^{100}\right)$ and $f$ are given in Fig. 1 .

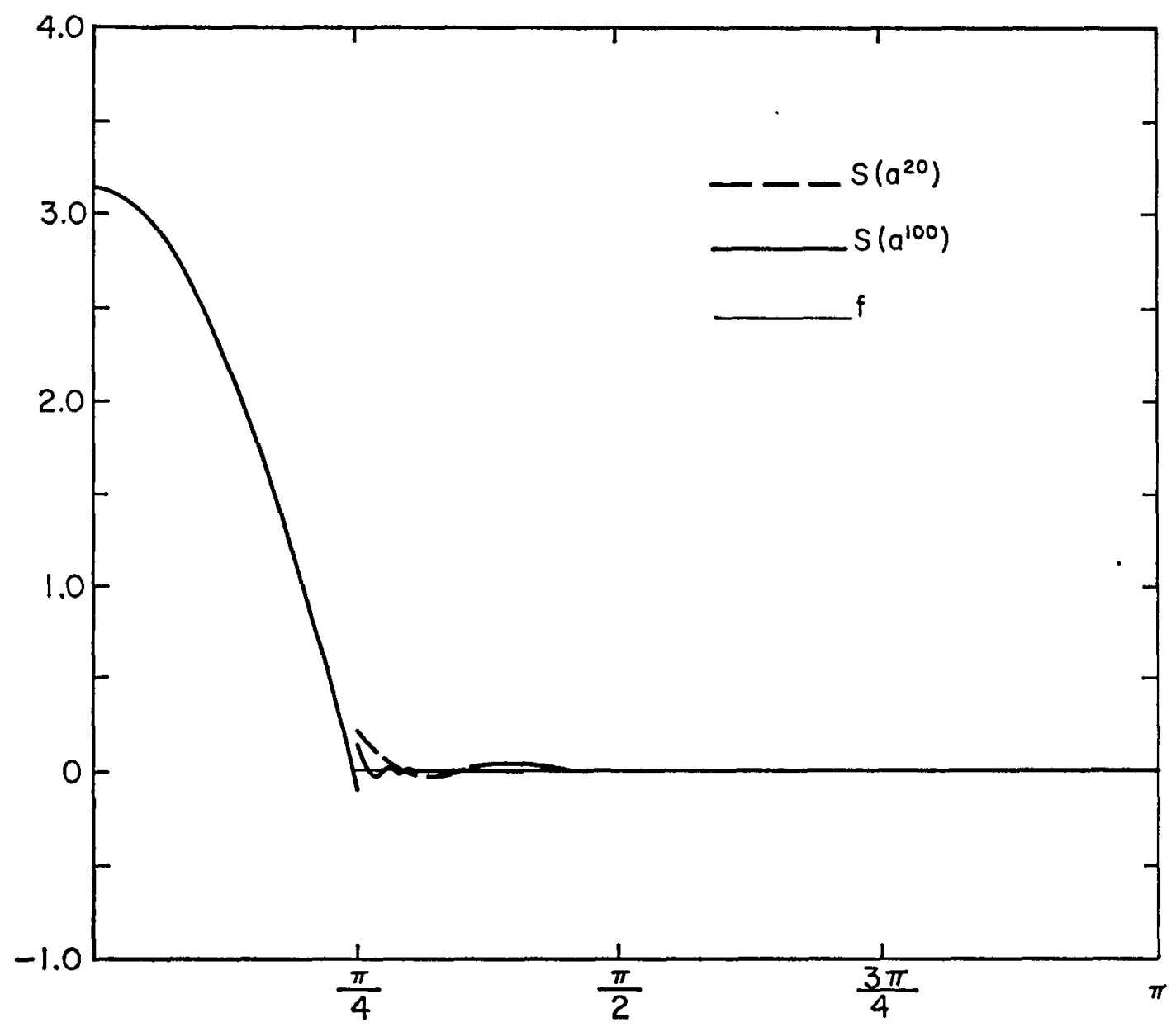

FIg. 1. Graphical illustration of Example 1. In the region $0<x<\pi / 4$ the fit between the approximate curves and $f(x)$ is so close that they appear indistinguishable on the graph. 
Example 2.

$$
\begin{aligned}
& \sum_{n=1}^{\infty} \frac{a_{n}}{n+\lambda-\frac{1}{2}}\left(\frac{2}{\pi}\right)^{1 / 2} \cos \left(n-\frac{1}{2}\right) x=1 \quad(0<x<\pi / 2), \\
& \sum_{n=1}^{\infty} a_{n}\left(\frac{2}{\pi}\right)^{1 / 2} \cos \left(n-\frac{1}{2}\right) x=0 \quad(\pi / 2<x<\pi) .
\end{aligned}
$$

Here $\lambda$ is a non-negative constant. This dual equation arises in seeking the potential $u$ in the rectangular strip $0<x<\pi$ and $y>0$ with the mixed conditions on $y=0: u=1$ for $0<x<\pi / 2$ and $u_{y}=\lambda u$ for $\pi / 2<x<\pi$. This problem illustrates the uniform manner in which least

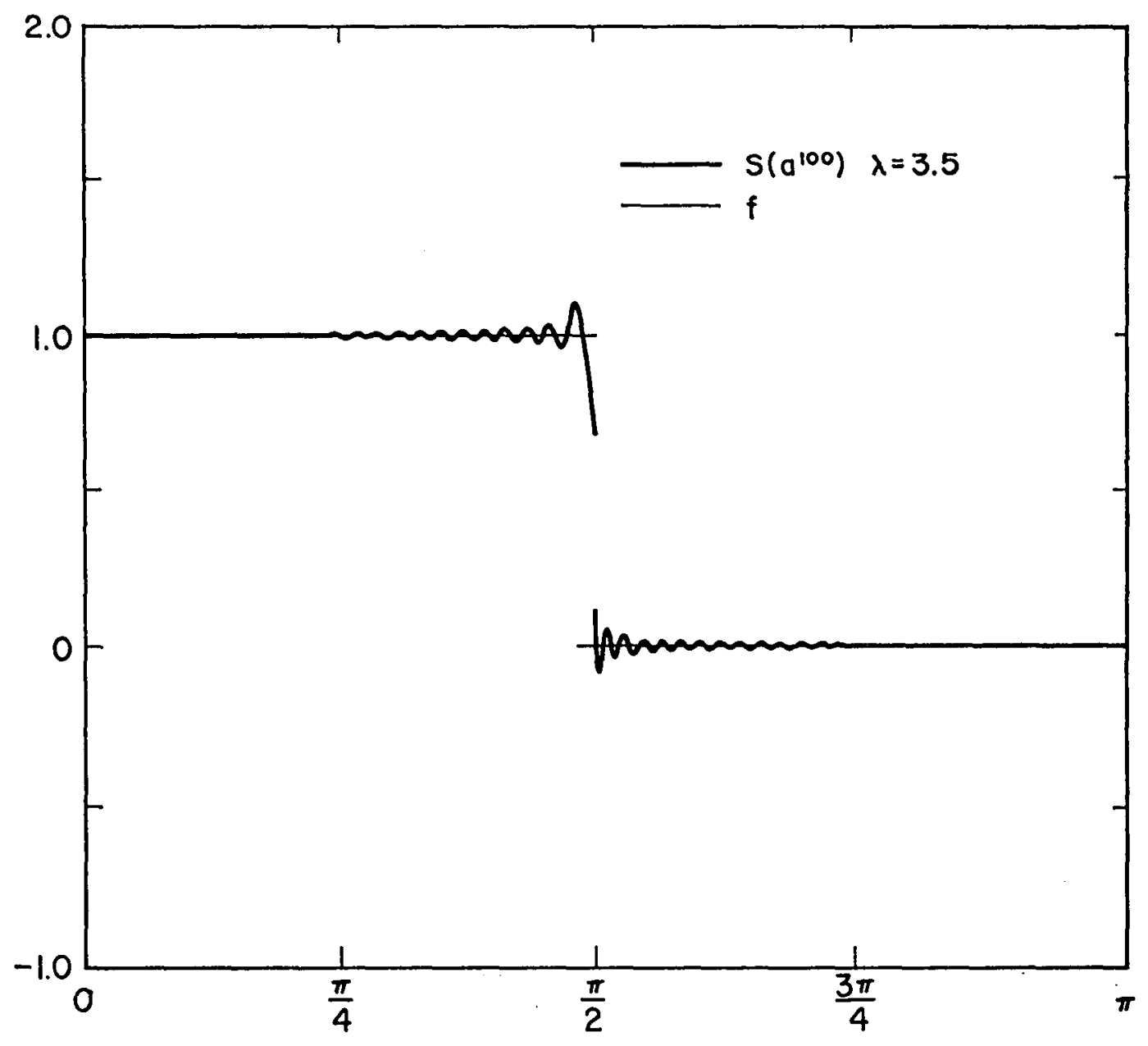

Fig. 2. Illustration of Example 2 with $\lambda=3.5$ showing the comparison between $f(x)$ and the 100th order approximation. 
squares analysis resolves dual trigonometric expansions arising in problems in which one of the mixed conditions is a radiation condition. Also, Tranter $[13,15]$ has given a closed form solution, not involving quadratures, for the Neumann condition $\lambda=0$, thus providing a useful test case. Tranter's solution is

$$
a_{n}=\left(\frac{\pi}{2}\right)^{1 / 2} \frac{P_{n-1}(0)}{K(\cos \pi / 4)},
$$

where $P_{n}$ is a Legendre polynomial and $K$ is the complete elliptic integral of the first kind. $S\left(a^{100}\right)$ and $f$ with $\lambda=3.5$ are shown in Fig. 2 , while in Fig. 3 the case $\lambda=0$ is illustrated using $S\left(a^{60}\right)$ and a 60-term Tranter approximation based on equation (6).

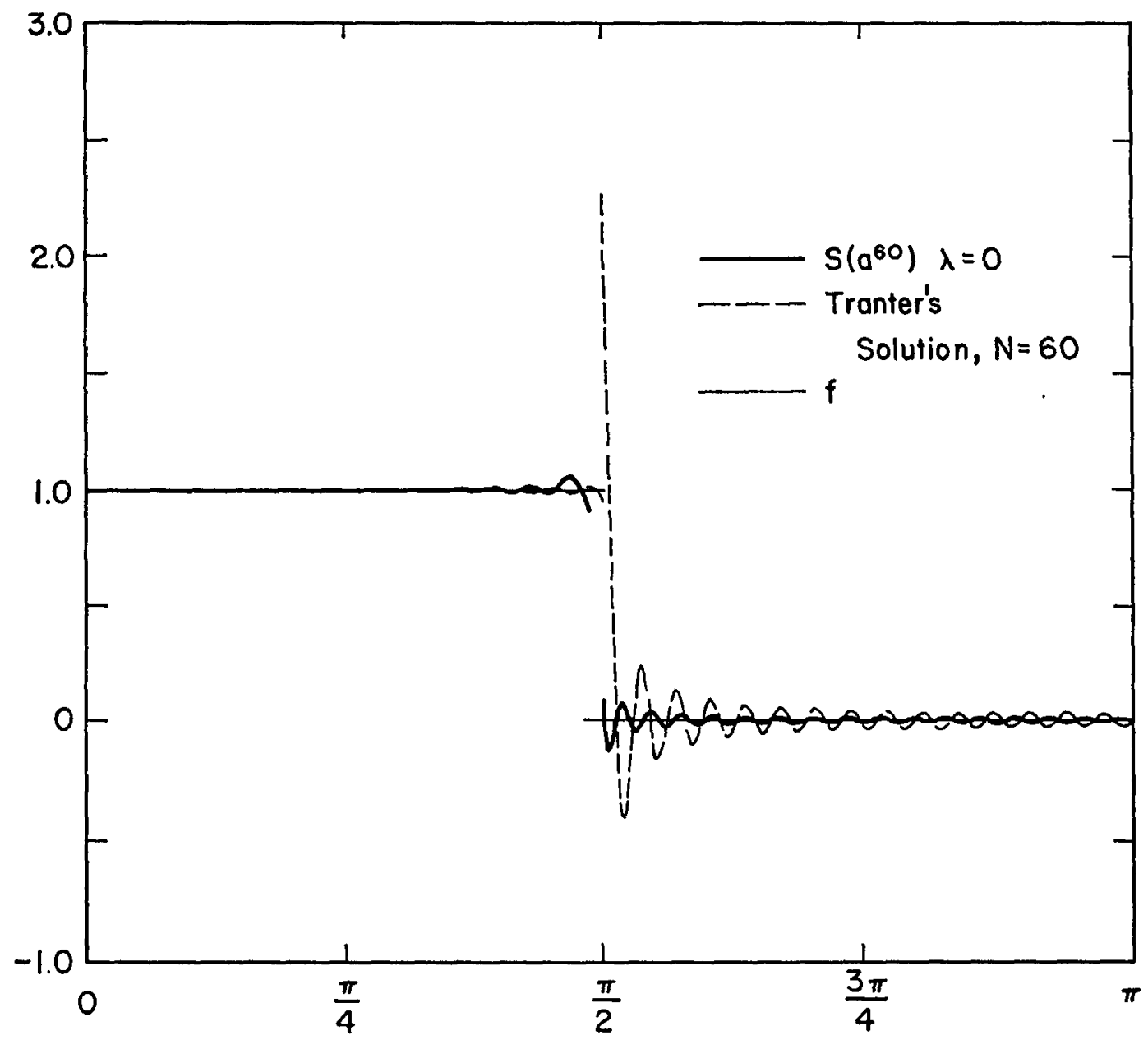

FIG. 3. Example 2 with $\lambda=0$ showing a comparison between the least squares approximation and the analytic solution given by equation (6). The two solutions are nearly the same for $0<x<\pi / 2$ while for $\pi / 2<x<\pi$ the least squares approximation gives what is clearly a closer fit. 
Example 3.

$$
\begin{aligned}
& \sum_{n=1}^{\infty} a_{n} n \tanh n \pi\left(\frac{2}{\pi}\right)^{1 / 2} \sin n x=\pi \quad(0<x<0.5), \\
& \sum_{n=1}^{\infty} a_{n}\left(\frac{2}{\pi}\right)^{1 / 2} \sin n x=x^{2} \quad(0.5<x<\pi) .
\end{aligned}
$$

This dual series tests least squares method with $\left\{\phi_{n}\right\}$ and $f$ chosen so that convergence must be slow and nonuniform (cf. [7, Section 16]); here $\phi_{n}(0)$ and $\phi_{n}(\pi)$ are both zero while neither $f(0)$ nor $f(\pi)$ is zero. This series arises in seeking the potential in the square $0<x, y<\pi$, with $u=0$ on three sides of the square, while on $y=0, u_{y}=-\pi$ for $0<x<0.5$ and $u=x^{2}$ for $0.5<x<\pi$. Results are shown in Fig. 4.

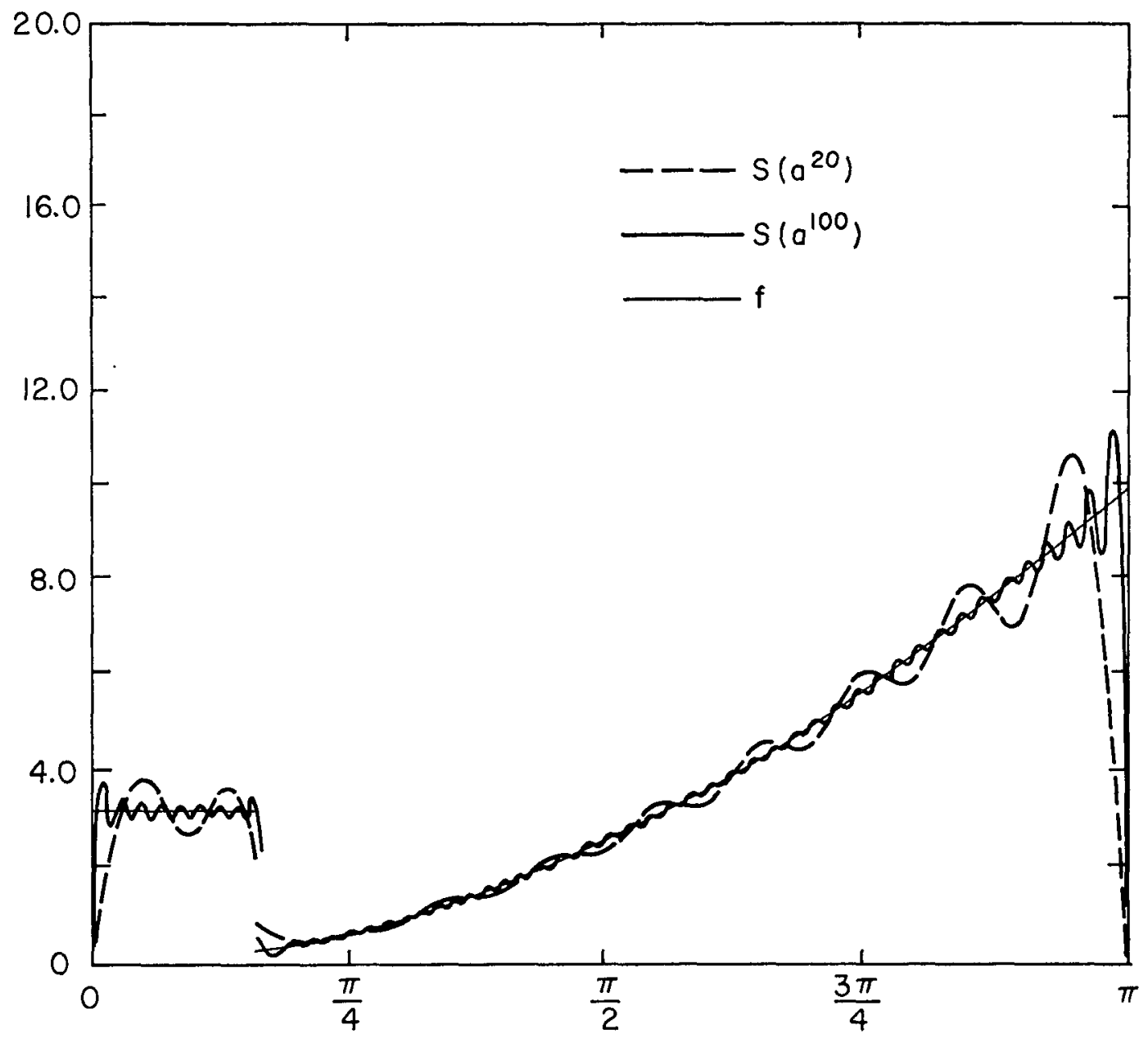

FIG. 4. This figure illustrates least squares approximations for Example 3. One observes a Gibbs' type phenomenon at the end points due to the choice of kernel. 
Example 4.

$$
\begin{aligned}
& \sum_{n=1}^{\infty} a_{n}\left\{1-\frac{\left(1+(-1)^{n}\right)}{4}\right\}\left[\frac{\pi}{2}+\frac{\sin ^{2} \lambda_{n} \pi}{2 \lambda_{n}^{2}}\right]^{-1 / 2} \sin \lambda_{n} x=\sin \left(\frac{\pi x}{e}\right) \quad(0<x<e / \pi), \\
& \sum_{n=1}^{\infty} a_{n}\left\{1+\left(1-(-1)^{n}\right)\right\}\left[\frac{\pi}{2}+\frac{\sin ^{2} \lambda_{n} \pi}{2 \lambda_{n}^{2}}\right]^{-1 / 2} \sin \lambda_{n} x=1 \quad(e / \pi<x<\pi),
\end{aligned}
$$

where $\lambda_{n}$ is the $n$th positive root of the equation $\lambda=-\tan \lambda \pi$. This example demonstrates a choice of modifier unrelated to applications and a kernel for which $\lambda_{n}$ is not an integer or an integer plus one half. Figure 5 presents plots of $S\left(a^{20}\right), S\left(a^{100}\right)$ and $f$.

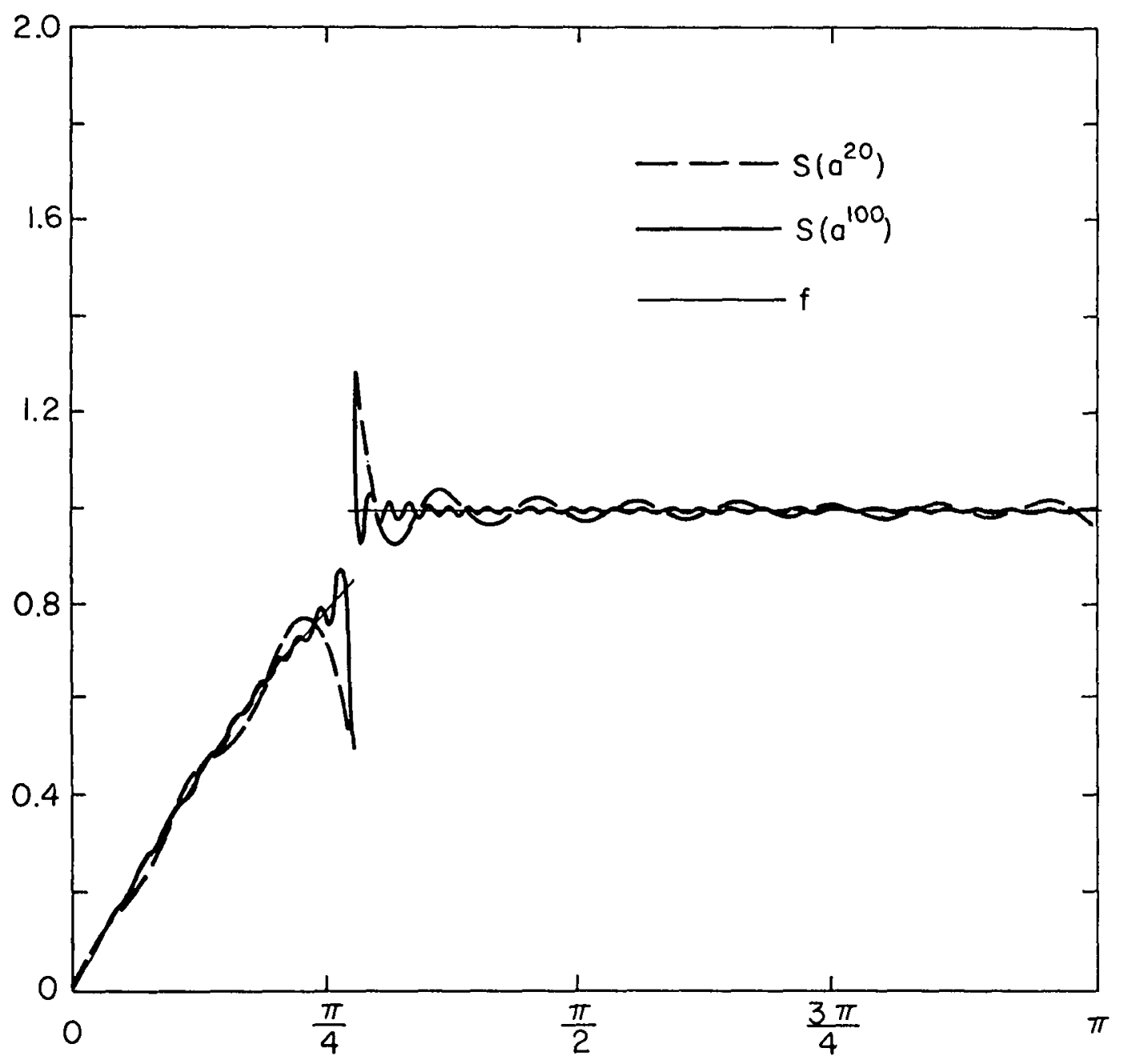

Fig. 5. The results for Example 4 based on 20th and 100th order approximations. 
5. Conclusion. To provide an indication of the acceptability of the answer given by equation (3), the relative least squares error $R(N)$ is introduced as

$$
R(N)=\frac{\left\|S\left(a^{N}\right)-f\right\|}{\|f\|} .
$$

TABLE I

\begin{tabular}{rcccccc}
\hline$N$ & \multicolumn{7}{c}{ Example } \\
& 1 & 2 & 2 & $2 *$ & 3 & 4 \\
\hline 20 & & $\lambda=3.5$ & $\lambda=0$ & $\lambda=0$ & & \\
40 & 0.020 & 0.065 & 0.024 & 0.23 & 0.22 & 0.036 \\
60 & 0.014 & 0.038 & 0.014 & 0.23 & 0.16 & 0.026 \\
80 & 0.011 & 0.031 & 0.011 & 0.23 & 0.13 & 0.023 \\
100 & 0.009 & 0.027 & 0.010 & 0.23 & 0.12 & 0.021 \\
& 0.008 & 0.024 & 0.009 & 0.23 & 0.11 & 0.021 \\
\hline
\end{tabular}

The relative least squares error computed from formula (7) for the examples discussed in Section 4. All error estimates are based on least squares solutions save for column $2 *$ where the analytic solution given by equation (6) was used.

Values of $R(N)$ for the examples given above are listed in Table I. These values and the above figures indicate that the least squares procedure has provided an effective approximation in each case.

\section{REFERENCES}

1. J. C. Cooke, Note on a pair of dual trigonometric series, Glasgow Math. J. 9 (1968), 30-35.

2. J. C. Cooke, The solution of some integral equations and their connections with dual integral equations and series, Glasgow Math. J. 11 (1970), 9-20.

3. P. J. Davis and P. Rabinowitz, Numerical integration (Waltham, Massachusetts, 1967).

4. E. L. Ince, Ordinary differential equations (New York, 1956).

5. V. V. Klyuyev and N. I. Kokovkin-Shcherbak, On the minimization of the number of arithmetic operations for the solution of linear algebraic systems of equations, Vycisl. Mat. i Mat. Fiz. 5 (1965), 21-33.

6. B. A. Kudriavtsev and V. Z. Parton, Dual trigonometric series in crack and punch problems, Prikl. Mat. Meh. 33 (1969), 844-849.

7. C. Lanczos, Discourse on Fourier series (New York, 1966).

8. B. Noble and M. A. Hussain, Exact solution of certain dual series for indentation and inclusion problems, Int. J. Eng. Sci. 7 (1969), 1149-1161.

9. B. Noble and J. R. Whiteman, Solution of dual trigonometrical series using orthogonality relations, SIAM J. Appl. Math 18 (1970), 372-379.

10. B. Noble and J. R. Whiteman, The solution of dual cosine series by the use of orthogonality relations, Proc. Edinburgh Math. Soc. 17 (1970), 47-51.

11. I. N. Sneddon, Mixed boundary value problems in potential theory (Amsterdam, 1966).

12. R. P. Srivastav, Dual series relations III. Dual relation involving trigonometric series, Proc. Roy. Soc. Edinburgh, Sect. A, 66 (1964), 173-209.

13. C. J. Tranter, Dual trigonometric series, Proc. Glasgow Math. Assoc. 4 (1959), 49-57. 
14. C. J. Tranter, A further note on dual trigonometric series, Proc. Glasgow Math. Assoc. 4 (1960), 198-200.

15. C. J. Tranter, An improved method for dual trigonometric series, Proc. Glasgow Math. Assoc. 6 (1964), 136-140.

16. J. R. Whiteman, Treatment of singularities in a harmonic mixed boundary value problem by dual series methods, Quart.J. Mech. Appl. Math. 21 (1968), 41-50.

17. W. E. Williams, The solution of dual series and dual integral equations, Proc. Glasgow Math. Assoc. 6 (1964), 123-129.

Colorado State University

Fort COllins, COLORADO 80521, U.S.A. 